\title{
Qucs-0.0.19S: a new open-source circuit simulator and its application for hardware design
}

\author{
Mike Brinson \\ Centre for Communications Technology, \\ London Metropolitan University, UK, \\ e-mail: mbrin72043@yahoo.co.uk
}

\author{
Vadim Kuznetsov \\ Department of Electronic Engineering, \\ Bauman Moscow State Technical University, \\ Kaluga branch, Russia; \\ e-mail: ra3xdh@gmail.com
}

\begin{abstract}
Circuit simulation is widely used in communication 45 2 and control equipment hardware design tasks. This article intro- 46 3 duces an extended version of the popular Qucs circuit simulator 4 called Qucs-0.0.19S. It is a simulation tool which supports 5 multiple SPICE circuit simulators, including Ngspice and Xyce.

6 The package includes a graphical user interface, component and 49

7 compact device modelling tools, a choice of simulation engine,

8 and advanced simulation data postprocessing facilities. It allows

9 user to construct new component using XSPICE extension and

10 construct new simulations using Nutmeg scripting. Qucs-0.0.19S

11 is targeted at academic and industrial applications. Software

12 implementation details and application cases are considered.

Index Terms-Qucs, SPICE, Ngspice, Xyce, Nutmeg scripting, 5 14 circuit simulation, EDA
\end{abstract}

\section{INTRODUCTION}

16 17 terprise information technology. However, not all sectors have 60 eliminated, making Qucs-0.0.19S, a viable choice for research 18 a fully developed software base. One example is electronic de- 61 and industrial circuit design [9], [10].

19 sign automation (EDA) where General Public Licence (GPL)

20 circuit simulation and printed circuit board layout packages are ${ }^{62}$

21 undergoing rapid development. The "Quite universal circuit 63

II. AN OVERVIEW OF QuCS-0.0.19S COMPONENT MODELS

22 simulator" (Qucs) [1], [2] is one of a new breed of GPL circuit 64 of Qucs circuit schematics with Ngspice or Xyce launched ${ }_{23}$ simulators. Qucs was started by M. Margraf and S. Jahn in 65 as external simulation engines [11]. In general legacy Qucs ${ }_{24}$ 2001. The initial intention was that Qucs should be an RF 66 circuit doesn't require tweaking to simulate it with Qucs25 circuit analysis package which offered features not found in ${ }^{67} 0.0 .19 \mathrm{~S}$. Qucs legacy passive components can be simulated ${ }_{26}$ SPICE. Recently a new team took over responsible for Qucs 68 with Qucs-0.0.19S. In addition Qucs-0.0.19S introduces a 27 development.

${ }_{69}$ group of passive component models with SPICE format. Qucs ${ }_{28}$ Qucs-0.0.19S is a freely available package with versions for 70 legacy semiconductor device models are SPICE incompatible. ${ }_{29}$ Linux, Windows $\left(\odot\right.$ and MacOS $\odot$. It includes a simulation ${ }^{71}$ Similar to passive components active device models have a ${ }_{30}$ kernel called Qucsator. Although Qucsator has acceptable 72 fixed list of named parameters [1], [12]. Moreover, some of ${ }_{31}$ performance it is not fully compatible with SPICE $2 \mathrm{~g} 6$ or $3 \mathrm{f5} 73$ these are SPICE incompatible. Qucs-0.0.19S allows users to 32 [3], [4]. Qucs has a unique netlist syntax and model format 74 construct SPICE device definitions from a name, a model spec${ }_{33}$ with SPICE support implemented via a software compatibility 75 ifier and a SPICE style "modelcard". These can be attached 34 layer. It does not allow direct access to manufacturers SPICE 76 to a schematic symbol and passed directly to a SPICE kernel. 35 models and libraries. The compatibility layer also prohibits ${ }^{77}$ Qucs-0.0.19S subcircuit and library components form part 36 access to a number of SPICE built in models, simulation 78 of a file component subclass. These allow the construction of ${ }_{37}$ types and the Nutmeg scripting language. A "Spice4qucs" 79 more complex components from pre-defined model primitives 38 subsystem has been added to Qucs to form Qucs-0.0.19S 80 and manufactures models. Qucs-0.0.19S allows users access ${ }_{39}$ [5], and hence overcome these limitations. Qucs-0.0.19S was ${ }^{81}$ to the following types of file component:

40 presented during MOS-AK workshop at Graz, Austria [6]. $\quad 82$

41 Spice4qucs is not another SPICE simulation kernel but 83 42 acts as an interface to a number of established GPL SPICE 84 ${ }_{43}$ engines. These have excellent performance, but usually lack 85 44 a graphical user interface (GUI) for schematic capture and 86
1) Subcircuits, for the construction of new components from predefined components. This form of subcircuit is identical to the original Qucs implementation [12], except that each subcircuit is stored as a .SUBCKT netlist; 
${ }_{97}$ Algorithm 1 outlines the Qucs netlist building method. ${ }_{98}$ Qucsator does not use netlist sections [12]. A Qucs schematic ${ }_{99}$ is represented as a C++ class, consisting of a set of netlist pro100 cessing methods. A single method scans a schematic file in one ${ }_{101}$ pass and outputs information describing located components.

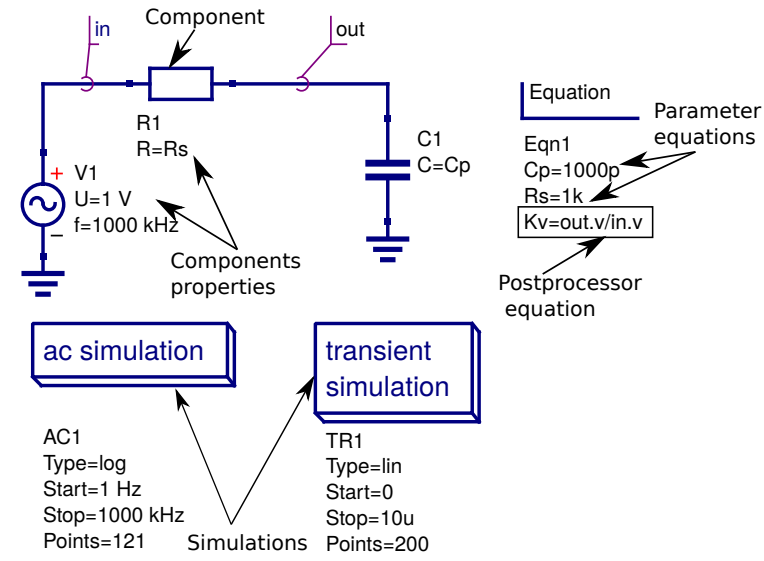

Fig. 1. A Qucs RC circuit schematic with netlist sections labelled

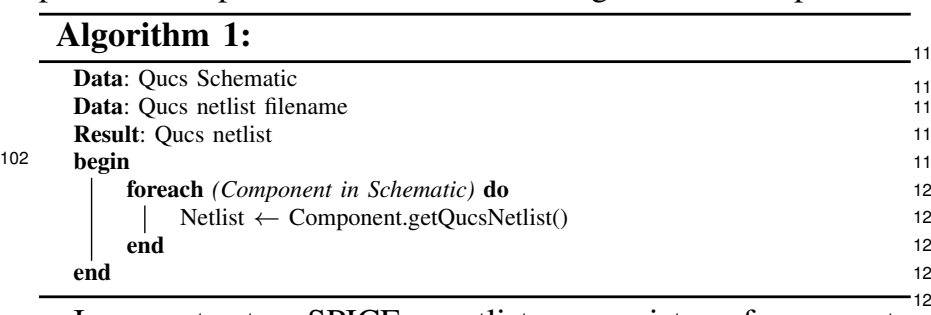
115 The Qucs netlist for the RC network is:
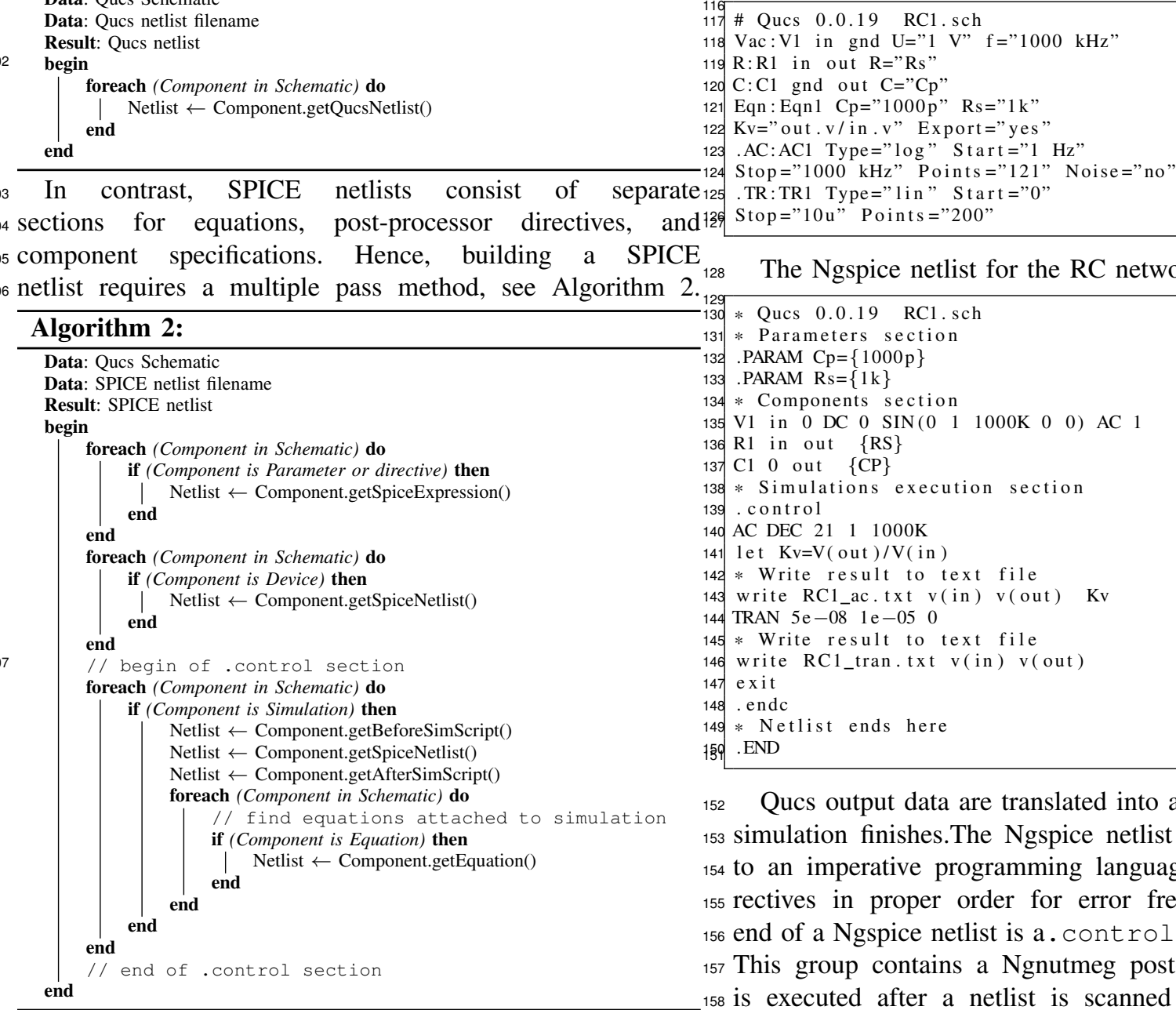

The Ngspice netlist for the RC network is:

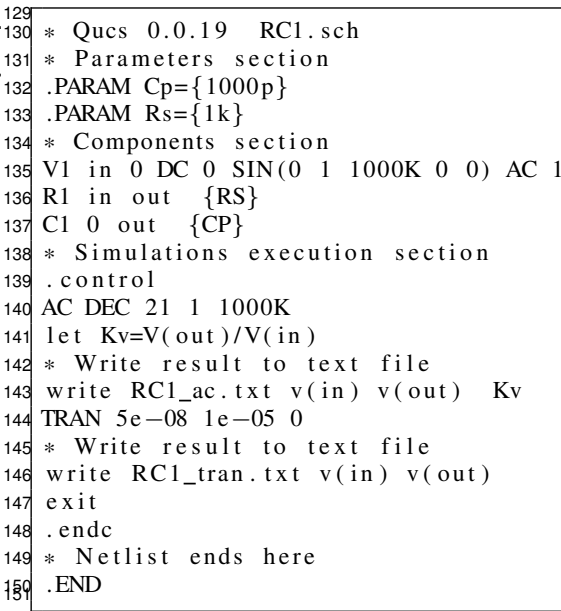

152 Qucs output data are translated into an XML dataset when 153 simulation finishes. The Ngspice netlist format is very close 154 to an imperative programming language, with . PARAM di155 rectives in proper order for error free evaluation. At the 156 end of a Ngspice netlist is a.control ... . endc group. ${ }_{157}$ This group contains a Ngnutmeg post-processor script that 158 is executed after a netlist is scanned by Ngspice. During 108 A Qucs schematic consists of a group of components where 159 scanning, simulation and post-processor directives are placed 109 every item has a properties list. For example, let's consider an ${ }_{160}$ between the control words .control ... .endc. The ${ }_{110}$ RC-network schematic (see Figure 11). Qucs simulation icons 161 . control ... . endc group also supports Ngnutmeg file 111 and equations are considered to be a special forms of compo-162 write directives for storing simulation datasets. Ngspice 112 nent. The Qucs netlist has declarative format. During scanning ${ }_{163}$ datasets are written in the SPICE-3f5 raw-ASCII format which ${ }_{113}$ Qucsator automatically separates components, equations, and ${ }_{164}$ in turn are converted and saved by Qucs-0.0.19S as part of a 114 simulator directives. The order has no effect on the final result. 165 Qucs XML dataset. 
166 With Xyce multiple simulations are not supported. The ${ }_{167}$ Xyce netlist has the following format:

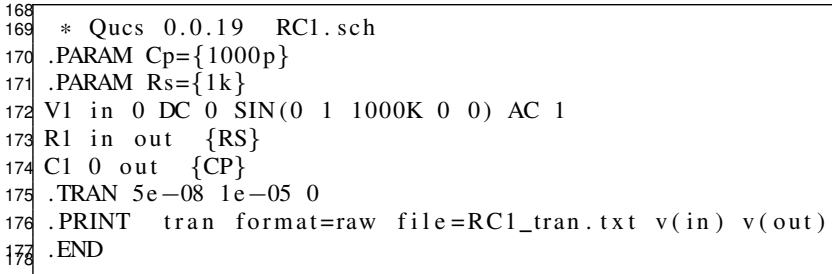

179 Spice4qucs operates at GUI level in distinct steps; netlist 180 building followed by simulation and finally it uses a raw${ }_{181}$ ASCII output data parser to generate a Qucs XML dataset. 182 All schematic symbols have an XML representation which is 183 written to memory during schematic file loading.

184 As the Xyce simulator does not include a data post185 processor the netlist building algorithm for Xyce is much 186 simpler, see Algorithm 3 .

187 The block diagram drawn in Figure 2 illustrates the in188 teraction between schematic capture, simulation and data 189 visualization for all used simulation backends.

190 A number of the SPICE simulation types generate Qucs 191 incompatible output datasets, implying that they require unique 192 custom parsers. The parsers implemented in the current ver193 sion of Qucs-0.0.19S are for SPICE-3f5 raw-ASCII (AC,214 to determine, apparent, active and reactive power, given by ${ }_{194}$ DC, TRAN, and Parameter sweep simulation), Fourier sim-215 $S=|U \cdot \bar{I}|, P=\Re[U \cdot \bar{I}], Q=\Im[U \cdot \bar{I}] .$, respectively. 195 ulation, noise simulation and HB simulation (XYCE only). ${ }^{216}$ Similarly, real power can be calculated from transient data, 196 The Spice4qucs subsystem extracts output data from each217 using $P(t)=u(t) \cdot i(t)$.

197 simulation request and combines them into single Qucs XML 198 dataset ready for processing by the Qucs data visualization 199 system.

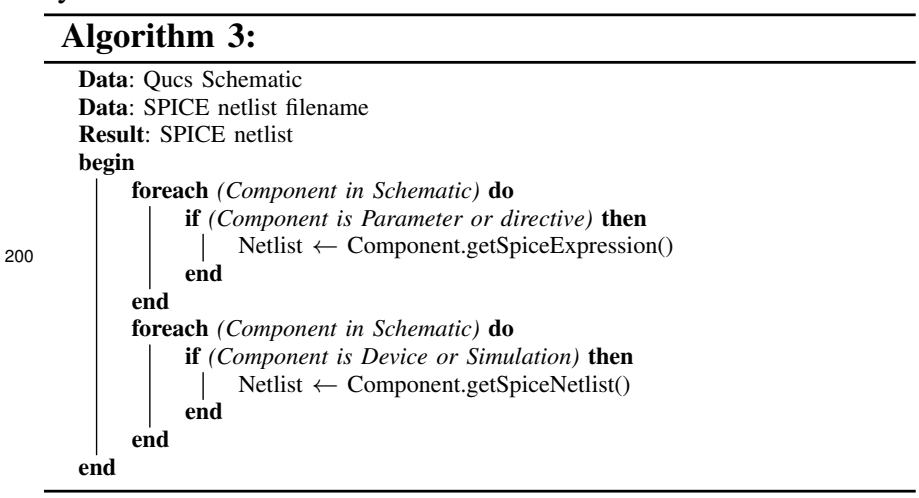

202 A. Common simulations and simulation data postprocessing

203 The following simulation types are implemented .DC, .AC, 204 .TRAN, .FOUR, .DISTO, .NOISE, and a new "Ngspice Cus205 tom" form. XYCE backend supports single-tone and multitone 206 Harmonic Balance simulation. Qucs allows to get access to 207 these simulations from the GUI.

208 The Qucs data post-processor has many SPICE incom209 patible functions. A way to overcome this is to pass post-218 B. Ngnutmeg scripting

210 processor directives directly to Nutmeg via a new component 219 Qucs-0.0.19S has a powerful new feature, called "Ngspice 211 called "Nutmeg equation". Illustrated in Figure 3 is an $\mathrm{RC}_{220}$ custom simulation", where a Nutmeg script is added to a Qucs 212 network driven by an AC source. This demonstrates how 221 schematic, allowing SPICE statements and Ngnutmeg scripts 213 .AC and .TRAN are defined and how "Nutmeg" can be used 222 to be passed directly to a SPICE netlist.

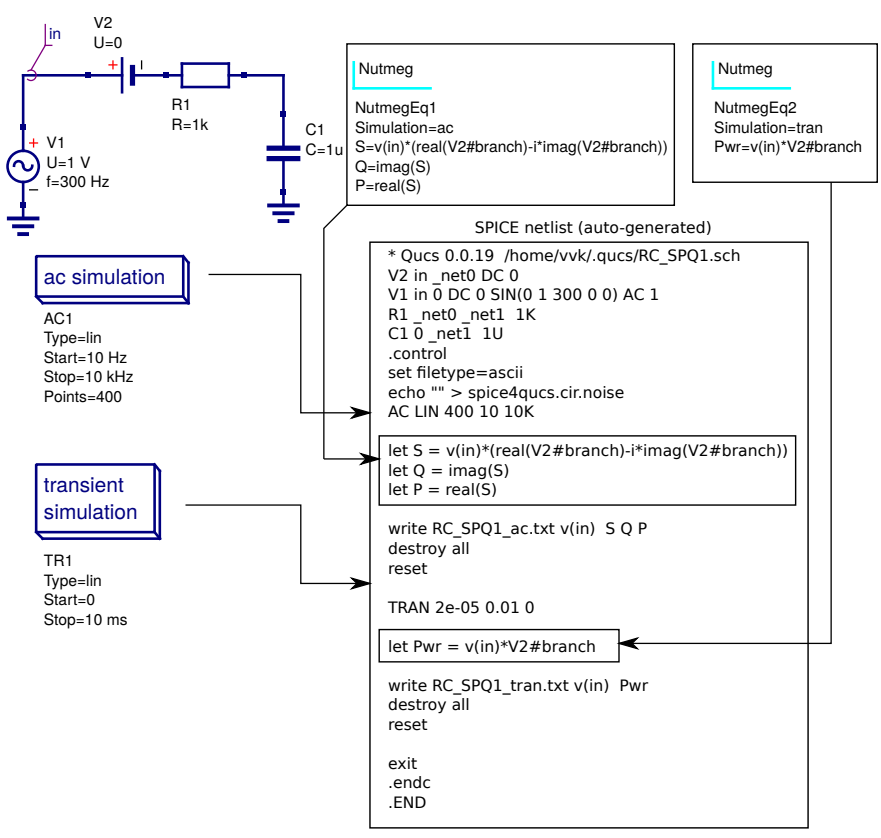

Fig. 3. An example of Nutmeg post-processor equation usage 
223 It allows to get easy access to all Ngnutmeg functions from 224 the GUI. It's able to construct nonstandard simulations using 225 Ngnutmeg scripting (for example scattering matrix and SWR 226 analysis, Monte-Carlo analysis).

227 For example, Z-parameter analysis is not available for the 228 most of SPICE-compatible simulators including proprietary 229 ones. But it could be easily constructed with Qucs-S, Ngspice, 230 and Nutmeg scripting. Figure 4 illustrates this approach for a ${ }_{231}$ passive low-pass Butterworth LC-filter.

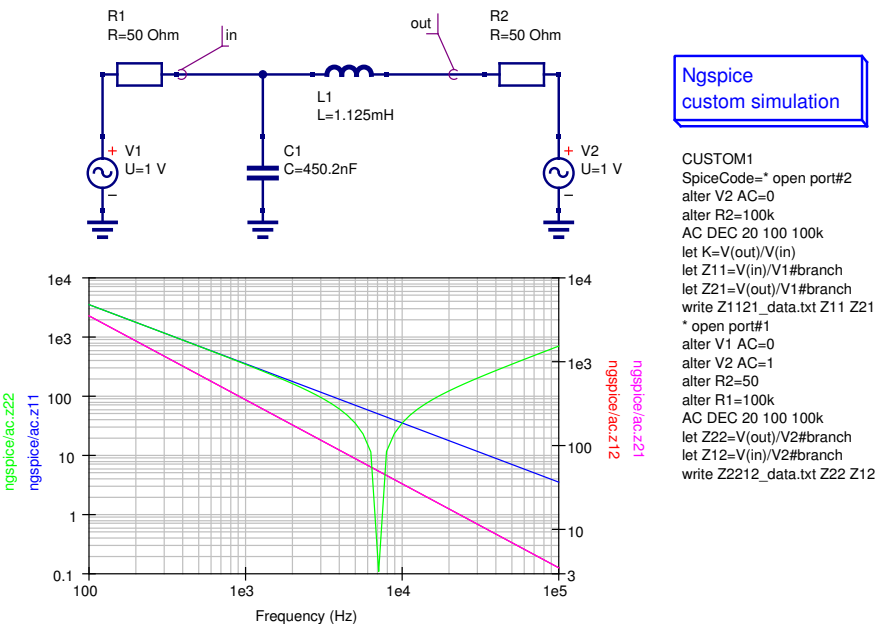

Fig. 4. Z-parameter extraction with Nutmeg scripting

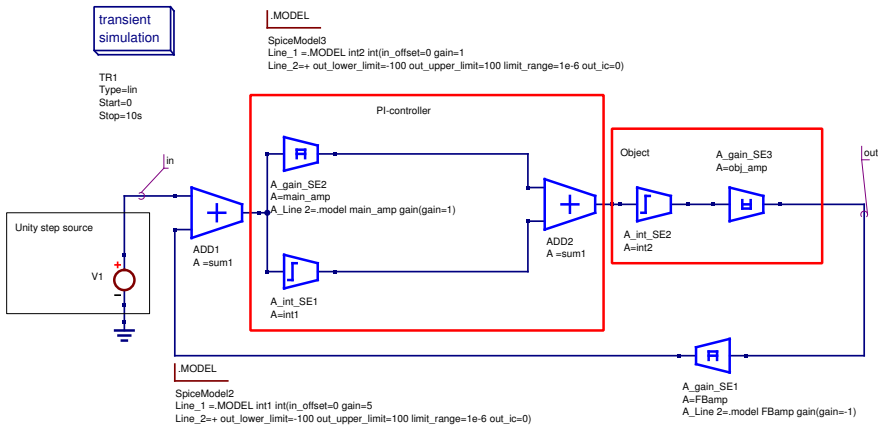

Fig. 5. PI-controller analysis with XSPICE analog blocks

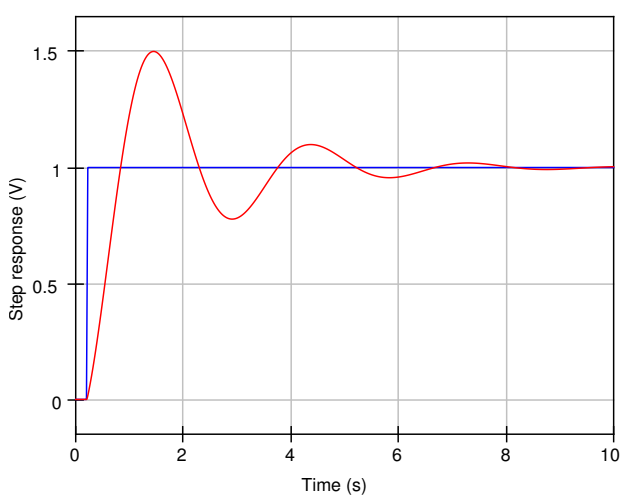

Fig. 6. Simulated step response of PI-controller
232 Postprocessor directives are used to extract voltage and 233 current data form AC-simulation results and convert it into ${ }_{234}$ desired Z-parameter value.

\section{V. XSPICE SUPPORT IN QUCS-S}

XSPICE is SPICE-3f5 extension targeted on system-level ${ }^{204}$ 237 circuit design tasks. It is especially important for commu- ${ }^{2}$ 238 nication equipment. XSPICE introduces a set of additional ${ }_{266}$ 239 analog and mixed-signal models targeted on system-level 240 design. Qucs-S with Ngspice backend supports a wide range ${ }^{267}$ 241 of XSPICE blocks.

${ }_{242}$ The following XSPICE analog devices are presented in ${ }_{243}$ Qucs-S out-of-box: gain block, integrator, differentiators, 244 adder, multiplier.

245 These blocks allows simulate not only analog circuits, but ${ }^{272}$ 246 also to solve control theory tasks. For example, PI-controller ${ }^{273}$ 247 step response analysis is shown in the Figure 5

${ }_{248}$ This simulation uses XSPICE blocks (analog gain, integra- ${ }^{275}$ 249 tor, and adder) to define PI-controller elements and transient ${ }^{276}$ 250 simulation to obtain step response.

277

251 It's able to construct a new XSPICE block using "XSPICE ${ }^{278}$ 252 generic device" component (Figure 7). It's sufficient to provide ${ }^{279}$ 253 port list and modelcard reference to create new device. It's ${ }^{280}$ 254 able to attach user symbol to a new device using standard ${ }^{281}$ ${ }_{255}$ Qucs subcircuit technique [12].

256 XSPICE allows to develop new devices using CodeModel ${ }^{283}$ 257 technique [13]. User can compile a set of CodeModels in ${ }^{284}$ 258 a single dynamic-loadable binary library. Now it's available 285 259 inclusion of precompiled CodeModel libraries using special 286 circuit symbol (Figure 7). It's sufficient to specify location of 61 binary library file. New models form this library could be used 262 using user-defined XSPICE block and general modelcard.

Qucs-S will allow to attach CodeModels to schematic and compile it automatically during netlist building. This feature is under construction now and it will not be considered further.

\section{CONCLUSION}

Qucs-0.0.19S is the first step in the development of an open8 source circuit simulator that combines, and extends, the best 9 features available with GPL circuit simulators. It can simulate a wide range of different size circuits, including those designed using manufacturer's device models.

Qucs-0.0.19S allows switching of simulation backends. Qucs-S covers the following application areas:

1) Realistic analog circuit simulation in time domain with Ngspice backend. Full support of SPICE-3f5 standard allows to use wide range of component models provided by vendors;

2) RF-circuits analysis (S,Z,Y-parameters matrix) using Nutmeg scripting and Harmonic balance analysis with XYCE backend [14]. This application is not available for many other SPICE-compatible simulators;

3) Control theory applications using XSPICE analog blocks;

The main advantages of Qucs-0.0.19S are:

1) It's free and open-source. It allows users to easily modify sources and propose new features; 

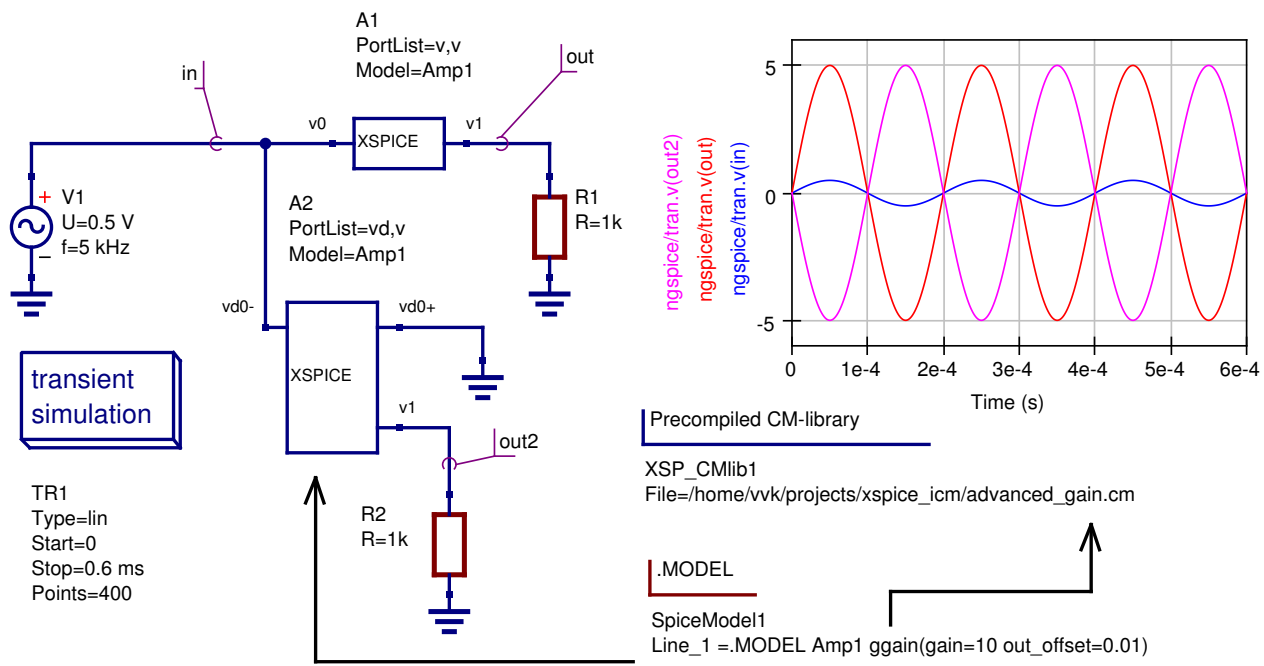

Fig. 7. User-defined XSPICE device construction 298 is not simple GUI for SPICE backends. It allows also ad-34 299 vanced features in simulation result postprocessing, circuit $_{343}^{342}$ 300 parametrization, and user devices and simulation definition. ${ }^{344}$ 301 And Qucs-0.0.19S could be recommended for communication ${ }^{345}$ 302 and control equipment equipment hardware design tasks.
2) Switchable simulation backends allows user to select the 326 most suitable one for every simulation task;

3) Advanced postprocessing with Nutmeg Equations;

4) GUI allows to get access to unlimited features of Nut- 330 meg scripting. It allows user to construct new simulation ${ }^{331}$ types (for example RF simulation types) without modi- ${ }_{333}^{332}$ fication of Qucs and simulator backends sources; 334

5) XSPICE allows system-level design. Also CodeModel ${ }^{335}$ technique allows to construct new XSPICE devices ${ }_{337}^{336}$ without modification of simulator sources.

338

0 [10]

[6] M. Brinson, R. Crozier, V. Kuznetsov, C. Novak, B. Roucaries, F. Schreuder, and G. B. Torri. Qucs: An introduction to the new simulation and compact device modelling features implemented in release 0.0.19/0.0.19Src2 of the popular GPL circuit simulator. MOSAK Workshop, Graz. [Online]. Available: http://www.mos-ak.org/graz_ 2015/presentations/T_5_Brinson_MOS-AK_Graz_2015.pdf

[7] Ngspice: mixed-level/mixed-signal circuit simulator based on Berkeley's Spice3f5. Ngspice project team. [accessed August 2015]. [Online]. Available: https://www.ngspice.org/

[8] Xyce Parallel electronic simulator: version 6.2. Sandia National Laboratories. [accessed August 2015]. [Online]. Available: https: //xyce.sandia.gov/

[9] A. Zonca, B. Roucaries, B. Williams, I. Rubin, O. D’Arcangelo, P. Meinhold, P. Lubin, C. Franceschet, S. Jahn, A. Mennella, and M. Bersanelli, "Modeling the frequency response of microwave radiometers with QUCS,” Journal Of Instrumentation, no. 5(12):T12001, November 2010.

0] V. Kuznetsov and L. Kechiev, "Charged Board Model ESD Simulation for PCB Mounted MOS Transistors," Electromagnetic Compatibility, IEEE Transactions on, vol. 57, no. 5, pp. 947-954, 2015.

[11] M. Brinson and V. Kuznetsov. Spice4qucs-help documentation. 346 User Manual and Reference Material. [Online]. Available: https: 347 //qucs-help.readthedocs.org/en/spice4qucs

348 [12] S. Jahn, M. Margraf, V. Habchi, and R. Jacob, Qucs. Technical papers. 349 [Online]. Available: http://qucs.sourceforge.net/docs/technical/technical. 350 pdf

351 [13] F. Cox III, W. Kuhn, J. Murray, and S. Tynor, "Code-level modeling in xspice," in Circuits and Systems, 1992. ISCAS '92. Proceedings., 1992 IEEE International Symposium on, vol. 2, May 1992, pp. 871-874 vol.2.

[14] M. Brinson and V. Kuznetsov, "Qucs equation-defined and Verilog-A RF device models for harmonic balance circuit simulation," in Mixed Design of Integrated Circuits Systems (MIXDES), 2015 22nd International Conference, June 2015, pp. 192-197. 